\title{
Experimental cosmic ray studies by the sub-array of the Alborz-I array
}

\section{S. Mortazavi Moghaddam, ${ }^{a}$ S. Abdollahi ${ }^{*},{ }^{b}$ M. Bahmanabadi ${ }^{b}$ and G. Rastegarzadeh ${ }^{a}$}

${ }^{a}$ Department of Physics, Semnan University

PO Box 35195-363, Semnan, Iran

${ }^{b}$ Department of Physics, Sharif University of Technology

PO Box 11155-9161, Tehran, Iran

E-mail:mortazavimoghaddamegmail.com abdollahi.soheiladgmail.com,

bahmanabadidsharif.edu grastegaresemnan.ac.in

The first phase of the Alborz Observatory Array (Alborz-I) is designed to have 20 scintillation detectors to study the cosmic ray spectrum in the energy range of $10^{12} \mathrm{eV}$ to $10^{16} \mathrm{eV}$. In order to collect data under stable environmental conditions, a sub-array consists of 5 plastic scintillation detectors on a pentagon with side of $5 \mathrm{~m}$ similar to the central cluster of the Alborz-I have been operationalized since February 2014. This paper studies angular distribution of detected showers and the angular resolution of the sub-array and compares it to the simulation result.

The 34th International Cosmic Ray Conference,

30 July- 6 August, 2015

The Hague, The Netherlands

\footnotetext{
* Speaker.
} 


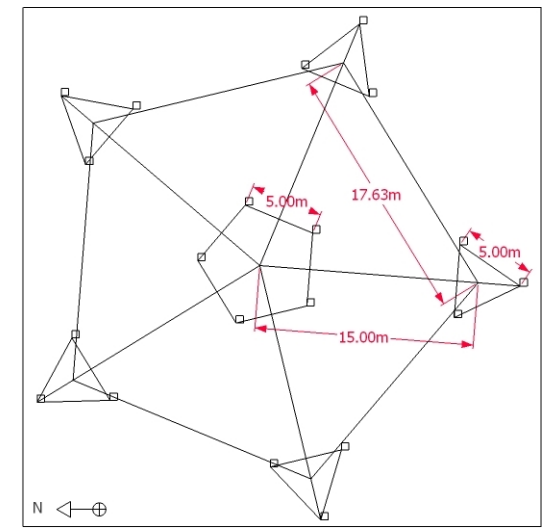

Figure 1: Layout of the Alborz-I array consist of 20 scintillation detectors.

\section{Introduction}

Study of the Extensive Air Showers (EASs) produced by interacting high energy cosmic rays with atmosphere, make it possible to find the characteristics of primary particles such as: mass, energy and arrival direction. Number and energy of the secondary particles are influenced by several parameters of atmosphere. Variation of atmospheric parameters like pressure and temperature can change the effective atmospheric depth in the shower trajectory and accordingly effect on the shower rate [四].

Ground base arrays is the most conventional method to detect and study the secondary particles by sampling them. Recording time differences of reached particles to detectors for an individual shower, enable us to estimate the arrival direction of incident primaries. So the angular resolution strongly depends on the timing accuracy which is affected by systematic uncertainties.

Alborz-I array consists of 20 scintillation detectors will be placed in a surface of $1600 \mathrm{~m}^{2}$ at Sharif University of Technology (SUT) campus at the altitude $1200 \mathrm{~m}$ a.s.l corresponding to the average atmospheric depth of $890 \mathrm{~g} / \mathrm{cm}^{2}$ and will be operationalized in near future (Fig. W). Preliminary steps that has been taken to prepare the Alborz-I array are: I) Optimizing the detectors [వ], II) Study on the array specifications including array layout, trigger condition, trigger efficiency, array response and angular resolution [3]. 5 scintillation detectors as a pentagon array, which is similar to the central cluster of the Alborz-I collects data in a stable atmospheric conditions since February 2014. The electronic devices of Alborz-I are VME modules and its software is being prepared, so ORTEC and CAEN units are used for this experiment.

This paper explains the experimental set-up and reveals obtained results of shower rate, the effect of atmospheric parameters on data, zenith angle distribution and angular resolution.

\section{Experimental Set-up}

The Alborz-I sub-array is set-up in the cosmic rays laboratory at $4^{\text {th }}$ floor of physics department of SUT under 2 concrete ceilings (each one with $40 \mathrm{~cm}$ thickness). In this sub-array 5 plastic scintillation detectors (each one with surface area of $50 \times 50 \mathrm{~cm}^{2}$ ) are placed at the corner of a pentagon with side length $5 \mathrm{~m}$. Each detector is embedded in a ironic pyramidal light enclosure with 


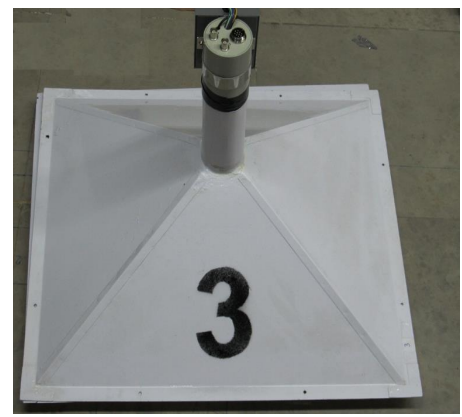

Figure 2: One of the scintillation detectors used in the experiment

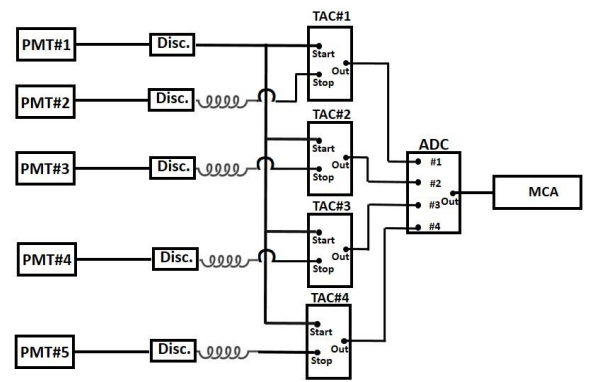

Figure 3: A schematic view of electronic circuit

$20 \mathrm{~cm}$ hight and a PhotoMultiplier Tube (PMT, 9813B) is installed at the apex of light enclosure (Fig. [2). It is worth mentioning that in the simulations [3] trigger condition of 5 out of 5 in the central cluster is one of the triggering conditions which obtains a good trigger efficiency and an apt angular resolution. So in analysing the experimental data the condition of all 5 fired detectors is enforced. To record data by this sub-array the coincidence method have been used. The schematic view of the used electronic circuit is illustrated in Fig. [1. The detectors are numbered from 1 to 5 and time lags of particles passing through the detectors No. 2, 3, 4, and 5 with detector No. 1 are recorded within a $200 \mathrm{~ns}$ time window. It should be noted that if there is a coincidence between detectors No. 1 and No. 2, other coincidences will be recorded.

Temperature and pressure of the laboratory have been checked during data acquisition and no dramatic change has been recorded, so the electronics operated in a stable atmospheric condition.

\section{Data Analysis}

The number of 131964 events have been recorded in 4788 hours of data collecting, that is, on average, one event in every $132.5 \mathrm{~s}$. The average number of events per hour within each month, and shower rate distribution are demonstrated in Figs. $\mathbb{t}$ and [1, respectively. The uniformity of counting rate of events during data recording is obvious in these two figures. In August and September, data acquisition was interrupted due to some technical problems. 


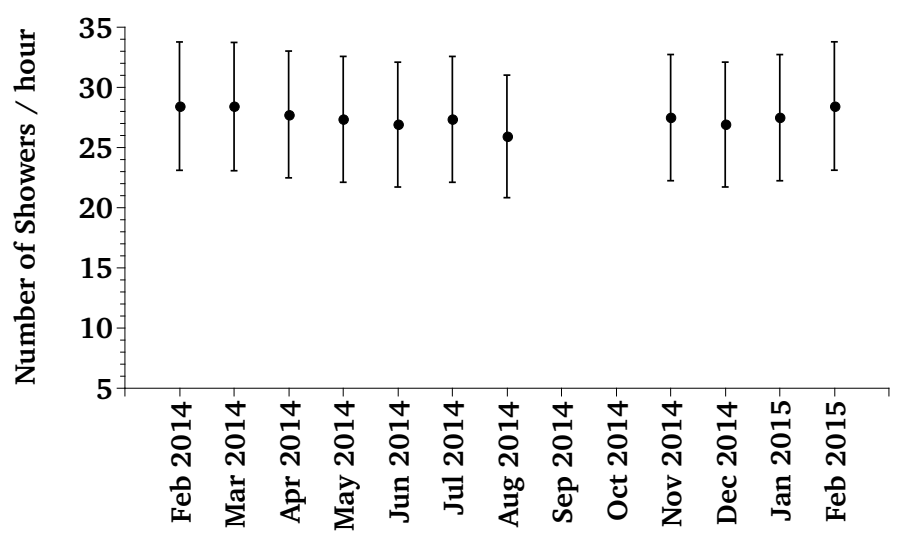

Figure 4: Average number of recorded events per hour within each month from February 2014 to February 2015.

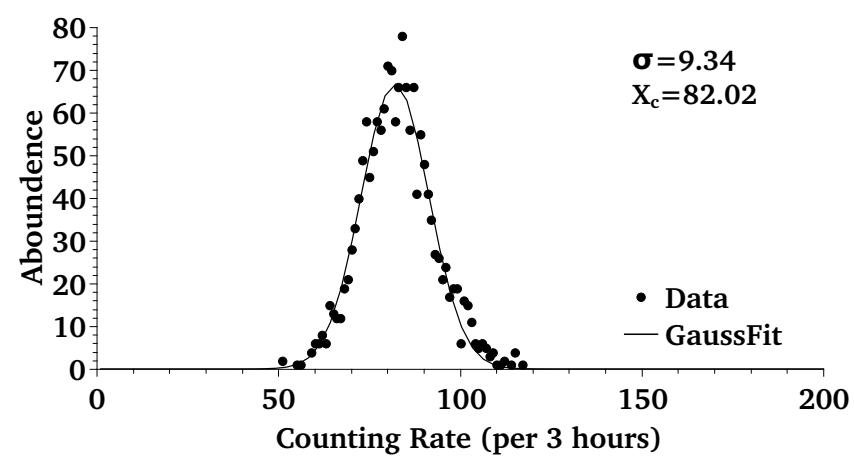

Figure 5: Counting rate distribution of events in 3 hour intervals.
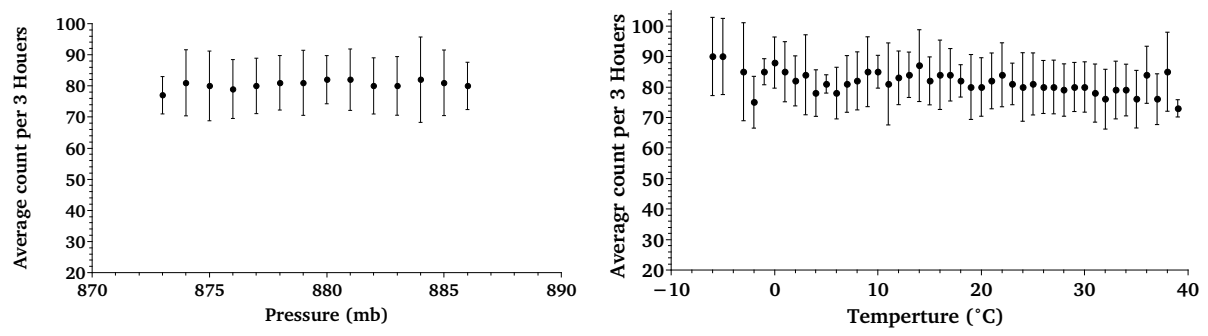

Figure 6: Average rate per 3 hours versus pressure(left) and temperature(right)

It can be inferred from figure $\$$ that with changing seasons, the rate remains rather invariant. In order to check the counting rate fluctuation with pressure and temperature variation, the average counting rate for each specific temperature and pressure during the first eight months of data recording is plotted in Fig. 6 . Temperature and pressure data was taken from Mehrabad Airport weather station which is close to SUT campus. As can be seen, there is no significant variation in counting rate by changing atmospheric conditions.

As shown in Fig Z, the zenith angle distribution of events is fitted by $\cos ^{n} \theta$ and the value of $\mathrm{n}$ is obtained $5.93 \pm 0.44$. The shower front is approximated by a plane and arrival direction 


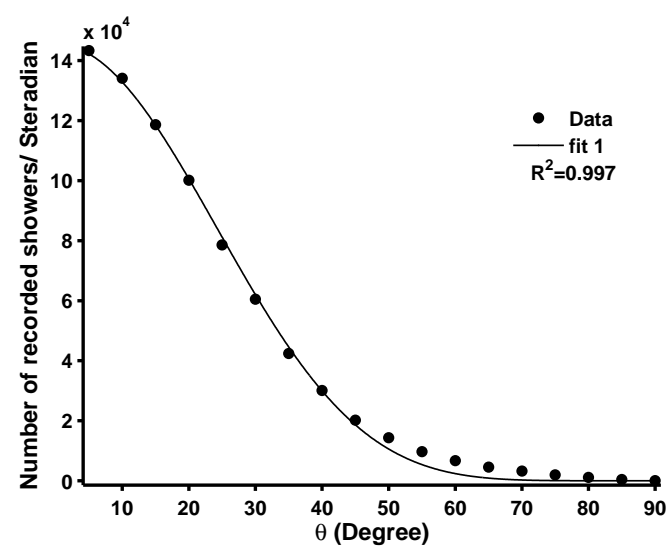

Figure 7: The zenith angle distribution.

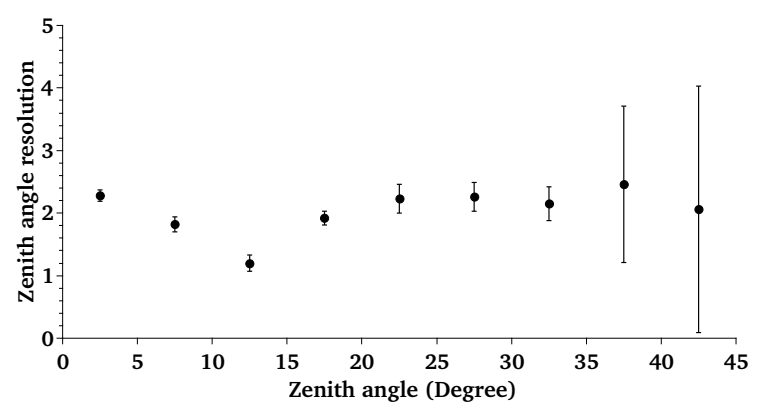

Figure 8: Angular resolution for trigger condition 5 out of 5 in the central cluster in the Alborz-I from simulation results.

\begin{tabular}{ccccc}
\hline Detectors & 1 and 2 & 1 and 3 & 1 and 4 & 1 and 5 \\
\hline HWHM (ns) & 4.17 & 5.62 & 5.37 & 6.60 \\
\hline
\end{tabular}

Table 1: HWHM of Gaussian function fitted to coincidence spectrum of experiments in order to measure the systematic error.

of primaries are calculated by minimizing chi-square method. The shower geometry, sub-array configuration and systematic uncertainties are result into minimum angular resolution of $13.31^{\circ}$ [团]. The systematic error of time measurement generated by the experiment instruments, including light enclosure and electronic devices, is obtained by a series of coincidence experiments. Detector No. 1 is inversely placed on a stand and each of other 4 detectors just seat on it respectively. Each coincidence spectrum is fitted with Gaussian function which their HWHM are reported in Table $\mathbb{W}$. Results obtained from simulation of the Alborz-I array shows that the zenith angle resolution for trigger condition 5 out of 5 in the central cluster of Alborz-I is less than $3^{\circ}$ (Fig. [ ا 8 ). This trigger condition is the same with the implemented trigger condition in the experiment, so the results can be compared. But the difference between simulation and experimental results is due to different timing accuracies which considered in their data analysis. As mentioned above, the old modules have been used in experiment cause a less accuracy in the timing while using VME modules will improve the timing accuracy and the results of simulation and experiment will match better. 


\section{Concluding Remarks}

An array similar to the central cluster of the Alborz-I array has collected data for about a year in stable atmospheric conditions in order to evaluate performance of the detectors. The effect of temperature and pressure on events rate have been studied. The results show that variation of temperature and pressure had no effect on the events rate and the uniformity of recorded events rate during data collecting can be a proof.

The zenith angle distribution of events obtains as $\cos ^{n} \theta$ with the value of $n=5.93 \pm 0.44$. The angular resolution of $13.31^{\circ}$ is estimated according to the systematic error which calculated from a series of coincidence experiments. The difference between results of experiment and simulation of the Alborz-I is due to different timing accuracy which applied in analysis processes. The results of simulation and experiment will become closer by using VME modules.

\section{References}

[1] Peter K.F. Grieder, Extensive Air Showers, Springer 2010

[2] Y. Pezeshkian et al., Scintillation detectors of Alborz-I experiment, Nucl. Instr. Meth. A 773 (2015) $117-123$

[3] S. Abdollahi et al., Alborz-I array: A simulation on performance and properties of array around the knee of cosmic ray spectrum, arXive:1501.00110V1

[4] M. Bahmanabadi et al., A study of the effect of geometric field on extensive air shower with small array Experimental astronomy 13 (2002) 39-57. 